Journal für Mobilität und Verkehr

ISSN 2628-4154

www.dvwg.de

\title{
Möglichkeiten und Grenzen der Analyse neuer Mobilitätsangebote mittels existierender Befragungen und neuartiger Erhebungsmethoden
}

\author{
Prof. Dr. Tobias Hagen, Nicole Reinfeld \\ siehe AutorInnenangaben
}

\begin{abstract}
Für die Verkehrsmodellierung sowie die Ableitung und Evaluation von Maßnahmen im Rahmen evidenzbasierter Verkehrspolitik und -planung werden unter anderem Befragungen als Datengrundlagen verwendet. Dieser Artikel stellt die existierenden Mobilitätsbefragungen in Deutschland vergleichend gegenüber und zeigt insbesondere mit Blick auf neue Mobilitätsangebote Lücken und Grenzen dieser Befragungen auf. Als Weiterentwicklung und Ergänzung werden Multimethodenansätze in Form der Kombination von Befragungs- und Tracking-Daten empfohlen.
\end{abstract}

Schlagwörter/Keywords:

Mobilitätsbefragungen, Neue Mobilitätsangebote, Stated Preferences, Tracking-Daten, Multimethodenansatz

\section{Einleitung}

Das Mobilitätsverhalten unterliegt dynamischen Veränderungen: Neue ergänzen und erweitern konventionelle Mobilitätsangebote oder treten gar mit ihnen in Konkurrenz (vgl. PTV/Fraunhofer ISI/M-Five 2019 sowie Barillère-Scholz et al. 2020). Neue Mobilitätsangebote können den Umstieg auf umweltfreundliche Verkehrsmittel fördern. Sie weisen dabei Schnittmengen mit den konventionellen Verkehrsmitteln auf. So zählen neben der geteilten Nutzung von Kleinstfahrzeugen wie E-Tretrollern, E-Scootern und (E-)Bikes auch Car-Sharing, Car-Pooling und Rideselling sowie öffentliche Bedarfsverkehre zu den neuen Trends, die zum Teil auf konventionellen Verkehrsmitteln wie dem Auto, dem Fahrrad oder Taxi aufbauen. Als ein Treiber führt die Digitalisierung dazu, dass die verschiedenen Mobilitätsarten durch die Errichtung von Mobilitätsstationen oder Online-Plattformen multimodal verknüpft werden können (vgl. ebd.). Darüber hinaus steigen - insbesondere getrieben durch technische Innovationen und jüngst die Corona-Pandemie - die Verkaufszahlen und die Nutzung von E-Bikes und Pedelecs (vgl. ZIV 2021), die das herkömmliche Fahrrad ergänzen bzw. zum Teil ersetzen und ebenfalls zu neuen Mobilitätsangeboten gezählt werden (vgl. Lanzendorf \& Hebsaker 2017).
Insgesamt scheint die Bedeutung des Fahrrads (inkl. E-Bikes etc.) mit der Pandemie gewachsen zu sein, was zu Herausforderungen für die Modellierung sowie Verkehrsplanung führt (vgl. bspw. Hagen et al. 2021).

Welchen Anteil neue Mobilitätsangebote am Modal Split ausmachen, ist jedoch nicht hinreichend erforscht. Aktuelle Daten - differenziert nach Region und verhaltenshomogenen Gruppen - sind für die Analyse und Modellierung neuer Mobilitätsangebote sowie für die Ableitung und Evaluation verkehrlicher Maßnahmen notwendig. Aktuell dienen neben Zählungen auch nationale Mobilitätsbefragungen als Datengrundlage (vgl. Dallmeyer 2014 sowie Hellenschmidt \& Wittwer 2007 und Friedrich 2011). Die für Verkehrspolitik und -planung bedeutendsten Befragungen sind "Mobilität in Deutschland“ (MiD), das „deutsche Mobilitätspanel“ (MOP) sowie „Mobilität in Städten“ (SrV) (vgl. Lanzendorf \& Schönduwe 2018).

Der Fokus dieser Befragungen liegt bisher auf der Kfz-Nutzung. Dies wird mitunter dadurch deutlich, dass bei der Befragung MiD neben der Kernbefragung, die bereits für jeden Haushalt Attribute der vorhandenen Autos (Hersteller, Modell, Jahresfahrleistung, Antriebsart, Baujahr bzw. 
Erstzulassung) enthält, noch weitere Module ${ }^{1}$ zum Autobesitz und detaillierteren Fahrzeugmerkmalen vorhanden sind (vgl. Eggs et al. 2018). Im MOP führen die Haushalte zusätzlich zur Erfassung der zurückgelegten Wege ein Tanktagebuch (vgl. Kantar 2020). Die übliche Nutzung von Wegen zu Fuß ist im Gegensatz dazu noch nicht in der Kernbefragung des MiD enthalten, sondern wird im Modul Nahmobilität und Radverkehr nur einem Teil der Befragten vorgelegt (vgl. Eggs et al. 2018). Um den thematischen Fokus über die KfzNutzung hinaus zu erweitern, werden zusätzliche Befragungen durchgeführt: So werden beispielsweise im „Fahrradmonitor" sog. „weiche“ Faktoren (vgl. Götz et al. 2016) wie Motive und Einstellungen von Radfahrerlnnen erfragt (vgl. Borgstedt et al. 2018). Neue Mobilitätsangebote wurden bislang nur vereinzelt und punktuell in den bestehenden Befragungen integriert. ${ }^{2}$

Das Ziel des Artikels ist es, Möglichkeiten und Grenzen existierender Befragungen für die Analyse neuer Mobilitätsangebote aufzuzeigen und neuartige Erhebungsmethoden zu skizzieren. Dazu werden im zweiten Kapitel typische Forschungsfragen im Hinblick auf neue Mobilitätsangebote dargestellt. Das dritte Kapitel gibt einen Überblick über die Adressaten bestehender Befragungen und die Zugangsmöglichkeiten zu den Rohdaten für Forschende und sonstige Nutzende. Anschließend werden im vierten Kapitel die bestehenden Befragungen vergleichend analysiert und darauf aufbauend Lücken aufgezeigt, die an zwei Beispielen verdeutlicht werden. Kapitel fünf diskutiert grundsätzliche Grenzen von Befragungen und die Möglichkeiten neuartiger Erhebungsmethoden, bevor das sechste Kapitel die Analyse mit einer Zusammenfassung und Schlussfolgerung beendet.

\section{Forschungsfragen im Hinblick auf neue Mobilitätsange- bote}

Mit der Entstehung neuer Mobilitätsangebote geht die Notwendigkeit einher, diese in konventionelle Mobilitätsangebote zu integrieren sowie ihre Nutzung zu analysieren und zu prognostizieren. Unzureichend vorliegende Parameter zur Modellierung und Planung des Verkehrs stellen dabei ein Problem dar, das nicht nur auf kommunaler Ebene von praktischer Bedeutung ist (vgl. Löwa \& Gertz 2017), sondern auch in der wissenschaftlichen Literatur Einzug gehalten hat (vgl. Deffner 2018). Die relevantesten Forschungsfragen können dabei in zwei Bereiche unterteilt werden:

\footnotetext{
1 „Um die Befragten nicht durch einen zu umfangreichen Fragenkatalog zu belasten“, wurden zusätzliche Befragungsmodule konzipiert, die jeweils nur einem Teil der Befragten vorgelegt werden (Nobis \& Kuhnimhof 2018, S. 20).

2 Siehe Kapitel 4.2: Grundsätzlich ist nur die Nutzung des Pedelecs, Carund Bikesharings in die Befragungen MiD, SrV und MOP integriert. Der Fahrradmonitor geht vereinzelt auf neue Mobilitätsangebote im Bereich Radverkehr und elektrische Kleinstfahrzeuge ein.
}

Zum einen fehlen gesicherte Erkenntnisse über die Determinanten des Mobilitätsverhaltens unter Berücksichtigung neuer Mobilitätsangebote. Zum anderen entstehen Herausforderungen bei der Integration neuer Mobilitätsangebote in die Verkehrsmodellierung (vgl. Oostendorp et al. 2020). Für konventionelle Verkehrsmittel wie den Kfz-Verkehr und in deutlich geringerem Maße für den Radverkehr existiert aus wissenschaftlicher Perspektive eine Vielzahl spezifischer Daten, Modelle und Analysen, die sich mit einzelnen Einflussfaktoren befassen, wie u. a. Routenwahl (Wermuth et al. 2006), Sicherheitskenngrößen (Baier et al. 2013 sowie Baier 2009), Verkehrsmittelwahl (Knapp 2015), Fahrbahnoberfläche (Leszczynski 2018) oder Wettereinfluss (Wessel 2020).

Determinanten des Mobilitätsverhaltens unter Berücksichtigung neuer Mobilitätsangebote

Im Hinblick auf neue Mobilitätsangebote ist eine zentrale Frage, welche Faktoren die Modus- sowie Routenwahl beeinflussen. Potenzielle Determinanten sind bspw. das Angebot und die Qualität der Infrastruktur, Knotenpunkte, demographische und sozioökonomische Variablen, Einstellungen und Präferenzen sowie Wetter, Umwelt und Topographie. Zusätzlich ist es relevant zu wissen, ob es wesentliche Unterschiede zwischen den Verkehrsmitteln und den Reisezwecken (z. B. Alltags-/Pendel- vs. Freizeitverkehr) gibt. Obwohl dem Pendelverkehr bislang die größere Relevanz zugeschrieben wurde, steigt der Anteil der Wege, die für Freizeitverkehre zurückgelegt werden (vgl. Götz \& Stein 2018). Vor dem Hintergrund der dynamischen Veränderungen der Mobilität, ist es darüber hinaus entscheidend, abschätzen zu können, wie sensibel NutzerInnen auf Veränderungen im Angebotsnetz reagieren - etwa abgebildet in Form von Elastizitäten (vgl. Schad et al. 2020). Wenn die Determinanten identifiziert und Parameter zur Modellierung empirisch ermittelt wurden, kann die Integration der neuen Mobilitätsangebote in Verkehrsmodellen erfolgen.

\section{Herausforderungen bei der Integration in Verkehrsmodelle}

Im Zuge der Modellierung müssen die relevanten Einflussgrößen auf der Angebots- und Nachfrageseite erfasst werden (vgl. Leihs et al. 2014). Die der Nachfrageseite zugrundeliegenden Nutzenfunktionen müssen um neue Mobilitätsangebote erweitert werden, da in klassischen Verkehrsmodellen kein allgemein anerkanntes Vorgehen zur Abbildung des nichtmotorisierten Verkehrs besteht (vgl. Bracher 2016). Darüber hinaus wird im Status quo bei der Modellierung in der Regel von den Hauptverkehrsmitteln eines Weges ausgegangen und Intermodalität nicht berücksichtigt (vgl. Kagerbauer et al. 2015). Dazu gehört auch die Nutzenbewertung kurzer und langer Wege (vgl. Lange \& Malik 2019) unter der Berücksichtigung von Mikromobilität, der „Fortbewegung mit elektrisch motorisierten sowie 
nicht motorisierten Kleinst- und Leichtfahrzeugen" (Difu-Berichte 2021, S. 16). Für die Modellierung ist die Ermittlung einer Geschwindigkeitsverteilung inkl. Streuung (Varianz) ebenfalls relevant (vgl. Dallmeyer 2014). Die dynamische Entwicklung in der Realität beeinflusst die Prognoseeigenschaften von traditionellen Einflussgrößen auf Modusund Routenwahl, was eine Überprüfung und Erweiterung der Prognosemodelle notwendig macht. Durch die neuen Möglichkeiten bei der Wahl des Verkehrsmittels bedarf es außerdem einer Prüfung bestehender verhaltenshomogener Gruppen, die üblicherweise für die Modellierung der Verkehrsnachfrage unterstellt werden (vgl. PTV/Fraunhofer ISI/M-Five 2019 sowie Cyganski \& Justen 2007).

\section{Adressaten der existierenden Mobilitätsbefragungen und Zugangsmöglichkeiten}

Bei der Betrachtung existierender Befragungen ist zu berücksichtigen, dass sie von verschiedenen Adressaten verwendet werden: So nutzen neben VerkehrsplanerInnen und Ingenieurbüros, PolitikerInnen und öffentlichen AuftraggeberInnen wie Verkehrsverbünden auch Forschungsinstitute bzw. -abteilungen die Datengrundlage mit unterschiedlichen Fragestellungen und Zielsetzungen. Die Zugänge zu den Datensätzen sind je nach Nutzendengruppe unterschiedlich ausgestaltet. Die Datensatzpakete des MiD liegen beispielsweise anonymisiert als Datensatzpaket A (reduzierter Variablenbestand, Vergröberung soziodemographischer und räumlicher Variablen) oder faktisch anonymisiert als Datensatzpakete B1-B3 (unterschiedliche Detailtiefe der räumlichen und soziodemographischen Variablen) vor. Die anonymisierten Daten können von AuftraggeberInnen regionaler Vertiefungsstichproben, Hochschulen oder sonstigen Einrichtungen mit der Aufgabe unabhängiger wissenschaftlicher Forschung sowie weiteren Dritten, welche die Daten zu Forschungszwecken im Rahmen eines Projekts im öffentlichen Auftrag bzw. eines öffentlich geförderten Projekts benötigen, bestellt werden. Die faktisch anonymisierten Datensatzpakete sind nur letzteren beiden Nutzendengruppen zugänglich. Damit geht einher, dass sich je nach Adressat, Fragestellung und Datensatz die abgeleiteten Ergebnisse unterscheiden (vgl. Blechschmidt et al. 2005).

\section{Vergleichende Analyse existierender Befragungen}

Die folgende Analyse baut auf der Arbeit von Lanzendorf \& Schönduwe (2018) auf, die die Befragungen MiD, MOP und SrV mit dem Ziel, deren Stärken und Schwächen für die kommunale Verkehrsplanung abzuleiten, gegenübergestellt haben. Sie berücksichtigen dabei Charakteristika der Stichprobenziehung, soziodemographische Variablen sowie Variablen der Verkehrsmittelnutzung und zentrale Mobilitätskennziffern der Befragungen für die Stadt Frankfurt am
Main in den Jahren 2002 bzw. 2003 und 2008. Dieser Artikel aktualisiert teilweise die von Lanzendorf \& Schönduwe (2018) verwendeten Vergleichsdimensionen um die letzte Befragungswelle und erweitert um neue Mobilitätsangebote sowie weitere Vergleichsdimensionen. Darüber hinaus wird die Befragung „Fahrradmonitor" ergänzend einbezogen, um sog. „weiche“ Faktoren in den Befragungsinhalten zu berücksichtigen. Als Quellen für die Gegenüberstellung dienen die jeweiligen Ergebnisberichte der letzten Befragungswellen (Ecke et al. 2020, Nobis \& Kuhnimhof 2018, Gerike et al. 2020, Borgstedt et al. 2018) sowie die Methodenberichte (Kantar 2020, Eggs et al. 2018, Hubrich et al. 2019) und Fragebögen. Auffälligkeiten bei der Gegenüberstellung werden durch Fettdruck in den Tabellen gekennzeichnet.

\subsection{Gegenüberstellung}

Wie aus der Tabelle 1 deutlich wird, behandeln die Befragungen MiD, MOP und SrV die Strukturmerkmale und Mobilität von Personen und Haushalten in Deutschland. Dabei fokussiert das MOP als Längsschnittstudie auf die Entwicklungen im zeitlichen Verlauf, während MiD und SrV wiederholte Querschnitte darstellen und sich im räumlichen Fokus unterscheiden (vgl. Hellenschmidt \& Wittwer 2007). Der Fahrradmonitor thematisiert ein subjektives Stimmungsbild von FahrradfahrerInnen. Die größten Unterschiede zwischen den Befragungen sind beim Fahrradmonitor die fehlende Veröffentlichung des Fragebogens, die fehlende Möglichkeit, die Rohdaten für Forschungszwecke zu erhalten sowie die Eingrenzung auf Erhebungseinheiten zwischen 14 und 69 Jahren. Beim MOP ist aufgrund des PanelCharakters keine einmalige regionale Aufstockung möglich. Inhaltlich weiterreichende Vertiefungen können aufgrund der Nutzung von Fragebogenmodulen bei der Befragung MiD herausgestellt werden. In Tabelle 2 werden die Befragungen im Hinblick auf wichtige soziodemographische Angaben gegenübergestellt.

Bezüglich der soziodemographischen Angaben erfassen die Befragungen sehr ähnliche Merkmale. Der Migrationshintergrund ist ausschließlich bei der Befragung MiD und dort nicht bei der Papierversion enthalten. Darüber hinaus wird in sämtlichen untersuchten Befragungen nur das biologische Geschlecht über die Auswahl zwischen „männlich" und „weiblich" erhoben. Die Antwortmöglichkeit "divers" war in den letzten Erhebungswellen nicht vorgesehen. Die Berücksichtigung der Genderperspektive, „dass Frauen und Männer, aber auch Kinder/Jugendliche, Mobilitätseingeschränkte, Ältere, Menschen mit Migrationshintergrund in der Gesellschaft unterschiedliche Lebensbedingungen und Chancen vorfinden", ist dabei notwendig, um strukturelle Unterschiede in den Daten analysieren zu können (Stiewe \& Krause 2012, S. 322). 
Tabelle 1: Thema, Auftraggeber und grundlegende Methodik der Befragungen

\begin{tabular}{|c|c|c|c|c|}
\hline & MiD & MOP & SrV & Fahrradmonitor \\
\hline Thema & $\begin{array}{l}\text { Strukturmerkmale und Mobilität von } \\
\text { Personen und Haushalten }\end{array}$ & \begin{tabular}{|l} 
Strukturmerkmale und Veränderungs- \\
prozesse im Mobilitätsverhalten von \\
Personen und Haushalten
\end{tabular} & $\begin{array}{l}\text { Strukturmerkmale und Mobilität von } \\
\text { Personen und Haushalten }\end{array}$ & $\begin{array}{l}\text { Subjektives Stimmungsbild der } \\
\text { Radfahrenden in Deutschland }\end{array}$ \\
\hline Auftraggeber & $\begin{array}{l}\text { BMVI, als Aufstocker: Länder, } \\
\text { überregionale Verbände, Kreise, } \\
\text { Kommunen, Städte, } \\
\text { Verkehrsunternehmen und -verbünde }\end{array}$ & BMVI & $\begin{array}{l}\text { Länder, überregionale Verbände, } \\
\text { Kreise, Kommunen, Städte, } \\
\text { Verkehrsunternehmen und -verbünde, } \\
\text { Aufgabenträger }\end{array}$ & $\begin{array}{l}\text { BMVI, als Aufstocker: Länder, } \\
\text { Kommunen, Städte }\end{array}$ \\
\hline Repräsentativität & $J a$ & Ja & Ja & Ja \\
\hline Grundgesamtheit & Wohnbevölkerung in Deutschland & $\begin{array}{l}\text { Wohnbevölkerung in Deutschland mit } \\
\text { deutschsprachigem Haushaltsvorstand }\end{array}$ & $\begin{array}{l}\text { Wohnbevölkerung im jeweiligen } \\
\text { Untersuchungsraum }\end{array}$ & Wohnbevölkerung in Deutschland \\
\hline Erhebungseinheiten & Haushalt, alle Personen des Haushalts & $\begin{array}{l}\text { Haushalt, bis zu fünf Personen des } \\
\text { Haushalts }{ }^{5}\end{array}$ & Haushalt, alle Personen des Haushalts & \begin{tabular}{|l|} 
Personen zwischen 14 und 69 Jahren \\
\end{tabular} \\
\hline $\begin{array}{l}\text { Stichprobenumfang } \\
\text { (jeweils letzte Erhebung }{ }^{1} \text { ) }\end{array}$ & 316.361 Personen & 3.872 Personen & 186.832 Personen & 3.053 Personen \\
\hline Erhebungsmethoden & CATI, CAWI, PAPI, Proxyinterviews ${ }^{3}$ & Schriftlich-postalisch, online & Telefonisch, online, Proxyinterviews & Online \\
\hline Erhobene Mobilitätsarten & Alltagsmobilität plus Module & Alltagsmobilität & Alltagsmobilität & Hauptsächlich Fahrrad \\
\hline Periodizität der Erhebung & $\begin{array}{l}\text { Wiederholter Querschnitt (alle fünf } \\
\text { Jahre) }\end{array}$ & $\begin{array}{l}\text { Panelerhebung rotierende Stichprobe } \\
\text { (jährlich, wobei ein Haushalt max. } 3 \\
\text { Jahre befragt wird) }\end{array}$ & $\begin{array}{l}\text { Wiederholter Querschnitt (alle fünf } \\
\text { Jahre) }\end{array}$ & $\begin{array}{l}\text { Wiederholter Querschnitt (alle zwei } \\
\text { Jahre) }\end{array}$ \\
\hline $\begin{array}{l}\text { Zugänglichkeit der } \\
\text { Rohdaten }\end{array}$ & $\begin{array}{l}\text { Für Forschende, öffentliche Stellen, } \\
\text { kommerzielle Nutzung }{ }^{4}\end{array}$ & $\begin{array}{l}\text { Für Forschende, öffentliche Stellen, } \\
\text { kommerzielle Nutzung }{ }^{4}\end{array}$ & Für wissenschaftliche Nutzung ${ }^{6}$ & Ergebnisse $^{7}$ \\
\hline $\begin{array}{l}\text { Module und } \\
\text { Sonderauswertungen }{ }^{2}\end{array}$ & \begin{tabular}{|l|} 
Module (Reisen, Autobesitz, \\
Fahrzeugmerkmale, pers. \\
Mobilitätsmerkmale, Infrastruktur und \\
digitale Versorgung, Nahmobilität und \\
Radverkehr, Zufriedenheit und \\
Einstellungen, Etappen), regionale \\
Vertiefungen \\
\end{tabular} & $\begin{array}{l}\text { Jährliche Sonderauswertungen in } \\
\text { Ergebnisberichten }\end{array}$ & $\begin{array}{l}\text { Auftraggeber können vertiefende } \\
\text { Fragen beauftragen }\end{array}$ & $\begin{array}{l}\text { Jährlich Sonderfragen möglich, } \\
\text { Vertiefungsstichproben - } \\
\text { Sonderfragen und Auswertung nach } \\
\text { Regionen möglich }\end{array}$ \\
\hline Anmerkung & & & & Fragebogen nicht veröffentlicht ${ }^{8}$ \\
\hline
\end{tabular}

Module $=$ Fragenblocke, die nicht allen Befragten gestellt werden, um die Länge des Fragebogens nicht übermäßig zu verlängern. Sonderauswertungen = sowohl thematische Erweiterungen des Fragebogens Is auch vertiefende Stichproben und Fragen, die beauftragt werden können.

3 CATI = Computer Assisted Personal Interview, CAWI = Computer Assisted Web Interview, PAPI = Paper And Pencil Interview, Proxyinterview = Stellvertretende Angabe für anderes Haushaltsmitglied.

Kosten Kommerzielle Nutzung MiD 201720 TEUR, MOP 20 TEUR für Zeitreihe.

Kinder unter 10 Jahren erhalten kein eigenes Wegetagebuch.

Stadtspezifische Auswertungen und Datensätze nur nach Freigabe der jeweiligen auftraggebenden Institutionen.

Ergebnisbericht als PowerPoint-Präsentation für regionale Auftraggeber (vgl. Nationaler Radverkehrsplan 2019) - falls spezifische Forschungsfragen bestehen, bietet Sinus Sonderauswertungen an. Der Zugang zu den Rohdaten ist auf Anfrage nicht möglich.

8 Den Fragebogen für Forschungszwecke zu erhalten, wurde auf Anfrage negativ beantwortet.

Tabelle 2: Befragungsinhalte - Soziodemographische Angaben

\begin{tabular}{|c|c|c|c|c|}
\hline & MiD & MOP & SrV & Fahrradmonitor $^{6}$ \\
\hline Biologisches Geschlecht & vorhanden & vorhanden & vorhanden & vorhanden \\
\hline Alter & vorhanden & vorhanden & vorhanden & vorhanden \\
\hline Berufstätigkeit (ja/nein) & vorhanden & vorhanden & vorhanden & vorhanden \\
\hline Berufstätigkeit Umfang ${ }^{1}$ & vorhanden & vorhanden & vorhanden & nicht vorhanden \\
\hline Migrationshintergrund & z.T. vorhanden ${ }^{2}$ & nicht vorhanden & nicht vorhanden & nicht vorhanden \\
\hline Bildungsabschluss & vorhanden & vorhanden & vorhanden & vorhanden \\
\hline Mobilitätseinschränkung & z.T. vorhanden ${ }^{3}$ & vorhanden & vorhanden & nicht vorhanden \\
\hline HH-Nettoeinkommen & vorhanden & vorhanden & vorhanden & vorhanden \\
\hline HH-Größe & vorhanden & vorhanden & vorhanden & nicht vorhanden \\
\hline Wohnort & Z.T. vorhanden ${ }^{4}$ & z.T. vorhanden 5 & z.T. vorhanden ${ }^{4}$ & z.T. vorhanden ${ }^{7}$ \\
\hline \multicolumn{5}{|l|}{$\begin{array}{ll}1 & \text { Voll- vs. Teilzeit. }\end{array}$} \\
\hline \multicolumn{5}{|l|}{2 Nicht im PAPI. } \\
\hline \multicolumn{5}{|l|}{$3 \quad$ Nicht für alle Befragten. } \\
\hline \multicolumn{5}{|c|}{4 Je nach Detailtiefe des Datensatzes. } \\
\hline \multicolumn{5}{|c|}{5 Postleitzahl/Gemeindekennziffer verkürzt. } \\
\hline \multirow{2}{*}{\multicolumn{5}{|c|}{$\begin{array}{ll}6 & \text { Fragebogen nicht veröffentlicht, Angaben beziehen sich auf den Ergebnisbericht. } \\
7 & \text { Größenkategorie des Wohnorts im Ergebnisbericht. }\end{array}$}} \\
\hline 7 Größenkategorie des $\mathrm{V}$ & & & & \\
\hline
\end{tabular}

Die Gemeinsamkeiten und Unterschiede der Befragungen im Hinblick auf die Erhebung der Verkehrsmittelwahl und -nutzung werden in Tabelle 3 gegenübergestellt.

Bei der Erfassung von Daten zur üblichen Mobilität und Verkehrsmittelnutzung werden unterschiedliche thematische Schwerpunkte bei den Befragungen deutlich: Während bei den Befragungen MiD, SrV und MOP detaillierte Fragen zum $\mathrm{Kfz}$ gestellt werden und Vertiefungen wie Module (MiD), ein Tanktagebuch (MOP) oder Detailfragen (SrV) vorhanden sind, werden beim Fahrradmonitor insbesondere Detailfragen zum Fahrrad erfasst. In allen Befragungen wird die Verfügbarkeit und Nutzungshäufigkeit der Verkehrsmittel Kfz, ÖPNV und Radverkehr erhoben. Die übliche Nutzungshäufigkeit von Wegen zu Fuß fragen explizit nur das MiD in einem Modul und der Fahrradmonitor ab.

Eine weitere Gegenüberstellung ist auf Basis des Wegetagebuchs in Tabelle 4 möglich. Der Fahrradmonitor wird in dieser Darstellung nicht berücksichtigt, da dort keine Erfassung 
Tabelle 3: Befragungsinhalte - Daten zu einzelnen Verkehrsmitteln und zur Verkehrsmittelwahl

\begin{tabular}{|c|c|c|c|c|}
\hline & MiD & MOP & SrV & Fahrradmonitor $^{1}$ \\
\hline PKW & $\begin{array}{l}\text { - Personenfragen: Verfügbarkeit, } \\
\text { Führerschein, Nutzungshäufigkeit } \\
\text { - Haushaltsfragen: Anzahl, Antriebsart, } \\
\text { Baujahr, Fahrleistung } \\
\text { - Modul Auto: Leistung, Halter, } \\
\text { Abstellmöglichkeit }\end{array}$ & $\begin{array}{l}\text { - Haushaltsfragen: Anzahl, Halter, } \\
\text { Nutzung (privat/ gewerblich), } \\
\text { Parkplatz, Parkmöglichkeiten } \\
\text { - Personenfragen: Verfügbarkeit, } \\
\text { Führerschein, Fahrgemeinschaften, } \\
\text { Parkplatzsituation am Arbeits- } \\
\text { /Ausbildungsplatz } \\
\text { - Tanktagebuch }\end{array}$ & $\begin{array}{l}\text { - Haushaltsfragen: Anzahl Privat-PKW, } \\
\text { Dienst-PKW, Fahrleistung, Antriebsart, } \\
\text { Zulassungsort, Parkplatz } \\
\text { - Personenfragen: } \\
\text { Verfügbarkeit, Führerschein, } \\
\text { Nutzungshäufigkeit }\end{array}$ & $\begin{array}{l}\text { - Haushaltsfragen: Verfügbarkeit } \\
\text { - Personenfragen: Nutzungshäufigkeit }\end{array}$ \\
\hline öV & $\begin{array}{l}\text { - Personenfragen: Nutzungshäufigkeit } \\
\text { ÖPNV, ÖPFV, Fernbus, Besitz Zeitkarte }\end{array}$ & $\begin{array}{l}\text { - Haushaltsfragen: Erreichbarkeit } \\
\text { Haltestellen zu Fuß, Zufriedenheit der } \\
\text { Anbindung an ÖV } \\
\text { - Personenfragen: Besitz Zeitkarte und } \\
\text { BahnCard separat, Erreichbarkeit } \\
\text { Arbeits-/Ausbildungsplatz mit ÖV } \\
\end{array}$ & $\begin{array}{l}\text { - Haushaltsfragen: Erreichbarkeit } \\
\text { Haltestellen zu Fuß } \\
\text { - Personenfragebogen: } \\
\text { Nutzungshäufigkeit, Besitz Zeitkarte }\end{array}$ & - Personenfragen: Nutzungshäufigkeit \\
\hline Fußverkehr & - Personenfragen: Häufigkeit ${ }^{2}$ & - Nicht explizit abgefragt & - Nicht explizit abgefragt & - Personenfragen: Häufigkeit \\
\hline Radverkehr & $\begin{array}{l}\text { - Haushaltsfragen: Anzahl } \\
\text { Elektrofahrräder und sonstigen } \\
\text { Fahrräder (funktionstüchtig) } \\
\text { - Personenfragen: Besitz Elektro- } \\
\text { fahrrad, Besitz Fahrrad ohne Elektro- } \\
\text { antrieb, Nutzungshäufigkeit allgemein, } \\
\text { Modul Radverkehr mit Detailfragen zu } \\
\text { Zugang, Sicherung, Helm }\end{array}$ & $\begin{array}{l}\text { - Personenfragebogen: Verfügbarkeit } \\
\text { von Elektrofahrrädern und sonstigen } \\
\text { Fahrrädern (ohne Elektroantrieb) }\end{array}$ & $\begin{array}{l}\text { - Haushaltsfragen: Verfügbarkeit von } \\
\text { betriebsbereiten Elektrofahrrädern } \\
\text { und sonstigen Fahrrädern (ohne } \\
\text { Elektroantrieb) } \\
\text { - Personenfragen: Nutzungshäufigkeit }\end{array}$ & $\begin{array}{l}\text { - Haushaltsfragen: Verfügbarkeit von } \\
\text { Fahrrädern ohne elektrische } \\
\text { Unterstützung, Elektrofahrrädern, } \\
\text { Pedelecs, Lastenrädern } \\
\text { - Personenfragen: Nutzungshäufigkeit } \\
\text { und -anlässe, genutzte Infrastruktur, } \\
\text { Helmbesitz und -nutzung, Pendeln, } \\
\text { Differenzierung Fahrradtypen }\end{array}$ \\
\hline Neue Mobilitätsangebote & $\begin{array}{l}\text { - Haushaltsfragen: Anzahl Personen } \\
\text { mit Carsharing-Mitgliedschaft } \\
\text { - Personenfragen: Carsharing- } \\
\text { Mitgliedschaft, Nutzungshäufigkeit } \\
\text { Carsharing, Nutzungshäufigkeit } \\
\text { Bikesharing }\end{array}$ & $\begin{array}{l}\text { - Personenfragebogen: Carsharing- } \\
\text { Mitgliedschaft }\end{array}$ & $\begin{array}{l}\text { - Personenfragen: Nutzungshäufigkeit } \\
\text { Carsharing, Nutzungshäufigkeit } \\
\text { Bikesharing }\end{array}$ & $\begin{array}{l}\text { - Personenfragen: Fragen zu Interesse, } \\
\text { Einsatz und Nutzung, } \\
\text { Einsatzmöglichkeiten von Pedelecs, } \\
\text { Bekanntheit, Nutzung, Kaufpotenzial } \\
\text { von Lastenrädern, Interesse an } \\
\text { Lastenradverleihsystemen, } \\
\text { Bekanntheit, Verbreitung, } \\
\text { Nutzungshäufigkeit von Bikesharing, } \\
\text { Kaufpotenzial von } \\
\text { Elektrokleinstfahrzeugen }\end{array}$ \\
\hline $\begin{array}{l}\text { Sog. „weiche" Faktoren } \\
\text { wie Motive und } \\
\text { Einstellungen }\end{array}$ & $\begin{array}{l}\text { - Personenfragen Modul Zufriedenheit } \\
\text { und Einstellungen: Bewertung } \\
\text { allgemeine Verkehrssituation vor Ort } \\
\text { sowie Einstellung für Verkehrsmittel } \\
\text { ÖPNV, Auto, Rad und Wege zu Fuß, } \\
\text { Modul Auto: Gründe für Nichtbesitz }\end{array}$ & $\begin{array}{l}\text { - Haushaltsfrage: Zufriedenheit der } \\
\text { Anbindung an ÖV }\end{array}$ & - Nicht verfügbar & $\begin{array}{l}\text { - Personenfragen: Beliebtheit } \\
\text { Verkehrsmittel, Gründe für Präferenz, } \\
\text { Nutzung in der Zukunft, sehr } \\
\text { detaillierte Fragen zu Verkehrspolitik, } \\
\text { Sicherheit, Nutzungsanlässe, } \\
\text { Fahrradpendeln, Fahrradstraßen, } \\
\text { Fahrradtourismus, Fahrradmarkt, Land } \\
\text { vs. Stadt }\end{array}$ \\
\hline
\end{tabular}

Kein Fragebogen veröffentlicht, Angaben beziehen sich auf den Ergebnisbericht.

Angaben bei Teil der Befragten mit Modul „Nahmobilität und Radverkehr" und in PAPI.

von Wegen vorgesehen ist.

Beim Vergleich der Daten, die mithilfe des Wegetagebuchs erhoben werden, ist insbesondere die Berichtsperiode ein Unterscheidungskriterium. Während im MOP aufgrund des Panelcharakters eine Kontinuität bei der Angabe zu zurückgelegten Wegen erforderlich ist, decken sowohl die Befragung MiD als auch SrV Stichtage im Laufe eines gesamten Jahres ab. Dabei ist zu berücksichtigen, dass die Erhebung im SrV nur mittlere Werktage (d. h. Dienstag bis Donnerstag) berücksichtigt. Zusatzstichproben für das Wochenende oder die gesamte Woche können von regionalen Auftraggebern initiiert werden.

Bei der Definition eines Weges nutzen die drei Befragungen dieselbe Definition und koppeln einen Weg immer an einen Wegezweck. Zum Beispiel können für den Wegezweck „Einkauf" in einer Mehrfachauswahl verschiedene Verkehrsmittel angegeben werden. Dagegen wird nicht erhoben, wenn ein Weg mehrere Zwecke hat. Darüber hinaus erfolgt keine Erfassung der einzelnen Etappen eines Weges, wenn mehrere Verkehrsmittel genutzt werden. Im Etappenmodul des MiD erfolgte 2018 diese Erfassung erstmals im Rahmen einer „regional disproportionale[n] und nicht repräsentative[n] experimentellen[n] Teilstichprobe" (Follmer 2019, S.6).

\subsection{Lücken existierender Befragungen an zwei Beispielen}

Basierend auf der vergleichenden Analyse der Mobilitätsbefragungen können (Daten-)Lücken an zwei Beispielen veranschaulicht werden.

\section{Berücksichtigung neuer Mobilitätsangebote}

Das erste Beispiel zeigt, dass neue Mobilitätsangebote bei der Befragung zur Verkehrsmittelwahl und -nutzung sowie der zurückgelegten Wege nur punktuell berücksichtigt werden. So erfassen

- MiD und MOP die Anzahl der Carsharing-Mitgliedschaften 
Tabelle 4: Befragungsinhalte - Mittels des „Wegetagebuchs“ erhobene Daten

\begin{tabular}{|c|c|c|c|}
\hline & MiD & MOP & SrV \\
\hline Berichtsperiode & $\begin{array}{l}\text { Jeweils ein Stichtag aus allen Tagen des } \\
\text { Erhebungszeitraums von } 12 \text { Monaten }\end{array}$ & $\begin{array}{l}\text { Wegetagebuch über sieben Tage als Vorgabe durch } \\
\text { Erhebungsinstitut (im Herbst) }\end{array}$ & $\begin{array}{l}\text { Jeweils ein Stichtag aus den Tagen Dienstag bis } \\
\text { Donnerstag innerhalb eines Jahres }\end{array}$ \\
\hline Definition von „Weg“ & $\begin{array}{l}\text { An einen Zweck gebunden unabhängig von } \\
\text { genutzten Verkehrsmitteln }\end{array}$ & $\begin{array}{l}\text { An einen Zweck gebunden unabhängig von } \\
\text { genutzten Verkehrsmitteln }\end{array}$ & $\begin{array}{l}\text { An einen Zweck gebunden unabhängig von } \\
\text { genutzten Verkehrsmitteln }\end{array}$ \\
\hline Erfassung der Wege & $\begin{array}{l}\text { - Alle Wege und Fahrten (auch z. B. kurze Wege und } \\
\text { Fußwege) } \\
\text { - Keine Erfassung einzelner Etappen (außer } \\
\text { Etappenmodul) }^{1} \\
\text { - Rundwege }{ }^{2} \text { können angegeben werden }\end{array}$ & $\begin{array}{l}\text { - Alle Wege und Fahrten (auch z. B. kurze Wege und } \\
\text { Fußwege) } \\
\text { - Keine Erfassung einzelner Etappen } \\
\text { - Rundwege erhalten eigenen Wegezweck bei } \\
\text { Plausibilisierung } \\
\end{array}$ & $\begin{array}{l}\text { - Alle Wege und Fahrten (auch z. B. kurze Wege } \\
\text { und Fußwege) } \\
\text { - Keine Erfassung einzelner Etappen } \\
\text { - Aufspaltung von Rundwegen }\end{array}$ \\
\hline Wegezwecke & $\begin{array}{l}10 \text { Zwecke mit weiteren } \\
\text { Detaillierungsmöglichkeiten im Anschluss }\end{array}$ & 9 Zwecke & $\begin{array}{l}20 \text { Zwecke die analog MID zusammengefasst } \\
\text { werden können }\end{array}$ \\
\hline Anzahl Wege & Bis zu 12 & Bis zu 51 & Alle Wege \\
\hline Verkehrsmittel & $\begin{array}{l}\text { Mehrfachauswahl aus } 20 \text { Verkehrsmitteln pro Weg } \\
\text { möglich: } \\
\text { - Zu Fuß } \\
\text { - Elektrofahrrad/Pedelec } \\
\text { - Fahrrad } \\
\text { - Moped/Mofa }{ }^{3} \\
\text { - Motorrad }{ }^{3} \\
\text { - Eigener o. anderer privater Pkw } \\
\text { - Carsharing-Fahrzeug }{ }^{4} \\
\text { - Lkw } \\
\text { - Stadtbus/Regionalbus } \\
\text { - U-Bahn/Stadtbahn } \\
\text { - Straßenbahn } \\
\text { - S-Bahn/Nahverkehrszug } \\
\text { - Anrufsammeltaxi, Rufbus o. ä. }{ }^{4} \\
\text { - Taxi } \\
\text { - Schiff/Fähre } \\
\text { - Fernzug (z. B. ICE, IC, EC) } \\
\text { - Fernbus im Linienverkehr } \\
\text { - Reisebus nicht im Linienverkehr }{ }^{4} \\
\text { - Flugzeug } \\
\text { - Anderes Verkehrsmittel }\end{array}$ & $\begin{array}{l}\text { Mehrfachauswahl aus } 12 \text { Verkehrsmitteln pro Weg } \\
\text { möglich } \\
\text { - Zu Fuß } \\
\text { - Elektrofahrrad/Pedelec } \\
\text { - normales Fahrrad } \\
\text { - Mofa, Moped, Motorrad } \\
\text { - Pkw als Fahrer } \\
\text { - Pkw als Mitfahrer } \\
\text { - Stadtbus/Regionalbus } \\
\text { - Fernbus/Reisebus } \\
\text { - U-Bahn/Stadt-/Straßenbahn } \\
\text { - S-Bahn/Regionalzug } \\
\text { - Fernzug } \\
\text { - Anderes, und zwar: }\end{array}$ & $\begin{array}{l}\text { Mehrfachauswahl aus } 20 \text { Verkehrsmitteln pro Weg } \\
\text { möglich } \\
\text { - Zu Fuß } \\
\text { - Fahrrad } \\
\text { - Elektrofahrrad } \\
\text { - Leih-/Mietfahrrad } \\
\text { - Moped/Motorrad/Motorroller } \\
\text { - Pkw-Fahrer im Haushalts-Pkw } \\
\text { - Pkw-Fahrer im Carsharing-Pkw } \\
\text { - Pkw-Fahrer im anderen Pkw } \\
\text { - Pkw-Mitfahrer im HH-Pkw } \\
\text { - Pkw-Mitfahrer im Carsharing-Pkw } \\
\text { - Pkw-Mitfahrer im anderen Pkw } \\
\text { - Bus } \\
\text { - Straßenbahn/Tram } \\
\text { - U-Bahn } \\
\text { - S-Bahn } \\
\text { - Nahverkehrszug } \\
\text { - Fernverkehrszug } \\
\text { - Fernbus } \\
\text { - Taxi } \\
\text { - Anderes: }\end{array}$ \\
\hline $\begin{array}{l}\text { Weitere Charakteristika } \\
\text { der Wege }\end{array}$ & $\begin{array}{l}\text { - Wochentag, Beginn Uhrzeit, Ankunft Uhrzeit, } \\
\text { ungefähre Strecke in km } \\
\text { - Start- und Endpunkt } \\
\text { - Mitfahrer oder Fahrer } \\
\text { - Begleitende Personen }\end{array}$ & $\begin{array}{l}\text { - Wochentag, Beginn Uhrzeit, Ankunft Uhrzeit, } \\
\text { ungefähre Strecke in km }\end{array}$ & $\begin{array}{l}\text { - Wochentag, Beginn Uhrzeit, Ankunft Uhrzeit, } \\
\text { ungefähre Strecke in km, } \\
\text { - Start- und Endpunkt sowie Kategorisierung } \\
\text { - Anzahl begleitender Haushaltsmitglieder und } \\
\text { anderer Personen } \\
\text { - Verkehrsmittel des längsten Teilstücks } \\
\text { - Umstiege bei ÖV } \\
\text { - Reihenfolge der Nutzung }\end{array}$ \\
\hline Sonstiges & $\begin{array}{l}\text { - Verfügarbeit Kraftfahrzeug am Stichtag } \\
\text { - Umfeld am Stichtag (verreist, gewohntes Umfeld) } \\
\text { - Außer Haus (ja/nein) } \\
\text { - Gründe für Immobilität } \\
\text { - Aufenthaltsort (z.B. Deutschland, nahes Ausland } \\
\text { etc.) } \\
\text { - Unterscheidung von regelm. beruflichen und } \\
\text { übrigen Wegen }\end{array}$ & $\begin{array}{l}\text { - Besonderheiten während des Wegetagebuchs (z.B. } \\
\text { Auto in Werkstatt, Urlaub, Krankheit, } \\
\text { Freitextangaben möglich) }\end{array}$ & $\begin{array}{l}\text { - Normaler Ablauf Stichtag (ja/nein) } \\
\text { - Gründe für Abweichung von normalem Ablauf (z.B. } \\
\text { Krankheit) } \\
\text { - Außer Haus (ja/nein) } \\
\text { - Gründe für Immobilität } \\
\text { - Verfügbarkeit PKW, Fahrrad, Elektrofahrrad, } \\
\text { Zeitkarte am Stichtag } \\
\text { - Untersuchungsraum am Stichtag (eigene } \\
\text { Gemeinde/Stadt oder anderes Gebiet) } \\
\text { - Detaillierung Zweck falls "Bringen, Holen" mit } \\
\text { weiteren Zielen (z.B. Grundschule) }\end{array}$ \\
\hline
\end{tabular}

der Person ${ }^{3}$,

- MiD und SrV die übliche Nutzungshäufigkeit von Carsharing der Person,

- Alle Befragungen die Verfügbarkeit eines Fahrrads mit elektrischem Antrieb ${ }^{4}$ und

- MiD, SrV und Fahrradmonitor die übliche Nutzungshäufig keit von Bikesharing der Person.

3 Im MiD zusätzlich Anzahl Mitgliedschaften im Haushalt. $4 \mathrm{MiD}$ : pro Person und Haushalt, MOP: pro Person, SrV: pro Haushalt, Fahrradmonitor: pro Haushalt.
Bei der Aufzeichnung einzelner Wege können im Wegetagebuch des MiD und SrV Carsharing-Fahrzeuge und beim SrV zusätzlich Leih- und Mietfahrräder angegeben werden. Alle drei Befragungen mit Wegetagebuch berücksichtigen das Elektrofahrrad/Pedelec als Verkehrsmittel. Darüber hinaus ist es beim MOP und SrV möglich, ein sonstiges, nicht gelistetes Verkehrsmittel mit Freitextangabe anzuführen. Die Konsistenz zwischen den Angaben zur üblichen Verkehrsmittelnutzung und des Wegetagebuchs wird dabei nur beim SrV gewahrt, da in beiden Frageblöcken Angaben zum Carsowie Bikesharing vorgesehen sind. Im MiD ist zusätzlich bei den CATI-/CAWI-Befragungen das Verkehrsmittel Anrufsammeltaxi, Rufbus o.ä. als Auswahlmöglichkeit vorgesehen. Weitere neue Mobilitätsangebote werden nicht abgefragt und sind nicht als Auswahlmöglichkeiten vorgesehen. 
Der Fahrradmonitor geht als Befragung mit einem abweichenden thematischen Fokus vereinzelt auf neue Mobilitätsangebote (und im weiteren Sinn innovative Entwicklungen) im Bereich Radverkehr und elektrische Kleinstfahrzeuge ein. So haben die Befragten die Möglichkeit, anzugeben, ob sie an Pedelecs interessiert, mit einem Pedelec gefahren oder bereits NutzerInnen sind. Darüber hinaus werden Nutzungszwecke und Gründe für fehlendes Interesse für Pedelecs erfragt. In Bezug auf Lastenräder werden die Bekanntheit, das Kaufpotenzial, Nutzungszwecke sowie im Sinne einer geteilten Nutzung das Interesse an Verleihsystemen erfasst. Die Fragen zum Bikesharing beschäftigen sich mit der Bekanntheit, der regionalen Verbreitung am Wohnort und der Nutzungshäufigkeit. Darüberhinausgehend haben die Befragten die Möglichkeit, Aussagen zu Lastenrädern und Bikesharing zu bewerten. Hinsichtlich elektrischer Kleinstfahrzeuge wird nur die Kaufabsicht in den nächsten zwölf Monaten thematisiert.

Somit erfolgt die Datensammlung zu neuen Mobilitätsangeboten in den dargestellten Befragungen, insbesondere im MiD, MOP und SrV, nur punktuell und nicht konsistent. Aussagen zur Nutzung, zum Modal Split und zu Motiven können basierend auf diesen Quellen nicht abgeleitet werden. Im Fahrradmonitor werden innovative Entwicklungen im thematischen Kontext des Radverkehrs ergänzend zu den bestehenden Mobilitätserhebungen erfragt. Für die Analyse verkehrlicher Maßnahmen und die Verkehrsprognose in der Forschung und Verkehrsmodellierung sind diese Quellen als Datenbasis für neue Mobilitätsangebote nicht ausreichend, während für die Abbildung des Kfz- und des öffentlichen Verkehrs eine breite Basis an Parametern vorliegt (vgl. Friedrich 2011). Weitergehend werden "weiche“ Faktoren wie Einstellungen und Motive benötigt (vgl. Döring \& Aigner-Walder 2017), da sie Mobilitätsmuster und -verhalten beeinflussen und in der Planung berücksichtigt werden sollten.

\section{Berücksichtigung sog. „weicher" Faktoren}

Dementsprechend stellt das zweite Beispiel die Verfügbarkeit von Daten zu „weichen“ Faktoren basierend auf der vergleichenden Analyse der Befragungen dar. So erfasst

- die Befragung MiD im Modul Zufriedenheit und Einstellun- gen die Bewertung der allgemeinen Verkehrssituation vor Ort sowie persönliche Einstellungen für die Verkehrsmittel ÖPNV, Auto, Rad und Wege zu Fuß sowie die Gründe für den Nichtbesitz eines Kfz-Fahrzeugs,

- das MOP die Zufriedenheit der Anbindung an den ÖV und

- die Befragung SrV keine weiteren Angaben zu Motiven und Einstellungen.

Der Fahrradmonitor berücksichtigt hingegen im Rahmen der thematischen Orientierung am Radverkehr eine Vielzahl von Fragen zu „weichen“ Faktoren wie die Beliebtheit von Verkehrsmitteln, Gründe für die Präferenz, Nutzungsabsichten in der Zukunft, die persönliche Einschätzung zur Verkehrspolitik, Nutzungsanlässe, Gefühl und Gründe für (Un-)Sicherheit, Motive und Rahmenbedingungen des Fahrradpendelns, Eindrücke zu verschiedenen Fahrradstraßen, zum Radtourismus etc.

Für den Einbezug neuer Mobilitätsangebote sind Kenntnisse über die tatsächliche Nutzung in Kombination mit den Anforderungen der Nutzenden notwendig (vgl. Klinger et al. 2016): Die Befragungen der letzten Befragungswellen weisen in beiden Bereichen - wie anhand der Beispiele dargestellt - Lücken auf, die für eine Beantwortung der in Kapitel 2 erörterten Forschungsfragen geschlossen werden müssen.

\section{Grenzen von Befragungen und neuartiger Ansätze}

Um die identifizierten Lücken in den Befragungsdaten zu schließen, sind neuartige Forschungsansätze erforderlich. Dabei ist zu berücksichtigen, dass in Befragungsdaten eine Diskrepanz zwischen potenziell für neue Mobilitätsangebote offenen und tatsächlichen Nutzenden festgestellt werden kann (vgl. Lanzendorf \& Hebsaker 2017): So geben Befragte an, sie wären bereit, neue Mobilitätsangebote zu nutzen, während sich in der tatsächlichen Nutzung zeigt, dass sie nur konventionelle Verkehrsmittel verwenden. Dies entspricht dem methodischen Unterschied zwischen der Messung von revealed preferences (RP) versus die Erhebung von stated preferences (SP). Tabelle 5 stellt die Methoden für unterschiedliche Erhebungsarten für Mobilitätsdaten

Tabelle 5: Revealed und Stated Preferences im Kontext von unterschiedlichen Methoden der Mobilitätserhebung

\begin{tabular}{|l|l|l|}
\cline { 2 - 3 } \multicolumn{1}{l|}{} & \multicolumn{1}{|c|}{ Revealed Preferences } & \multicolumn{1}{c|}{ Stated Preferences } \\
\hline Fragebogen & Datenerfassung: Wegetagebuch & $\begin{array}{l}\text { Datenerfassung: Hypothetischer Vergleich zw. Alternativen } \\
\text { Probleme: Unaufmerksamkeit, fehlende Attributausprägungen, Inkongruenz } \\
\text { mit RP (vgl. Danaf et al. 2019) }\end{array}$ \\
\hline Beobachtung/Tracking & $\begin{array}{l}\text { Datenerfassung: GPS-Tracking mit zusätzlichen Angaben zu Wegen und/oder } \\
\text { Personen }\end{array}$ & $\begin{array}{l}\text { Datenerfassung: Referenzszenario vs. hypothetische Alternativen (RP- } \\
\text { basierend) }\end{array}$ \\
& $\begin{array}{l}\text { Probleme: Technische Probleme, Genauigkeit GPS Tracking (Bierlaire et al. } \\
\text { 2010) }\end{array}$ & $\begin{array}{l}\text { Probleme: Repräsentativität (vgl. Weber \& Bauder 2013), Auswahl } \\
\text { Referenzszenario (vgl. Danaf et al. 2019) }\end{array}$ \\
\hline
\end{tabular}


gegenüber und zeigt mögliche Probleme auf.

Bei Untersuchungen zu RP geht es um Entscheidungen, die Einzelpersonen tatsächlich getroffen haben (vgl. Dell'olio et al. 2018). Die Fahrtenbücher, die im Rahmen der Befragungen MiD, SrV und MOP geführt werden, zeigen beispielsweise im Idealfall solche Entscheidungen bei der Verkehrsmittelwahl auf. Allerdings neigen Befragte in Wegetagebüchern dazu, kurze Wege zu vernachlässigen und die Reisedauern kurzer Wege zu überschätzen (vgl. Zhao et al. 2015 sowie Willumsen 2021). Darüber hinaus repräsentiert die Wahl eines Stichtags Personen unzureichend, deren Wege von Tag zu Tag stark variieren (vgl. Zhao et al. 2015). Weitere Nachteile von RP-Untersuchungen sind, dass sie nur tatsächlich bestehende Mobilitätsangebote widerspiegeln (vgl. Matyas \& Kamargianni 2017) und Probleme bei der statistischen Analyse wie hohe Korrelationen der unabhängigen (erklärenden) Variablen, fehlende Variablen oder mangelnde Variation der Variablen aufweisen (vgl. Vrtic 2005).

Experimente zu SP können einige der oben genannten Probleme lösen, indem den Befragten hypothetische Situationen bzgl. Verkehrsmittel oder Routen präsentiert werden (vgl. Dell'olio et al. 2018). Hypothetische Situationen werden durch ein Set aus Attributen charakterisiert und können Mobilitätsangebote beinhalten, die bisher nicht auf dem Markt existieren (vgl. Matyas \& Kamargianni 2017 sowie Willumsen 2021). Auch die Ausprägungen der Attribute können je nach dargestellter Situation so variiert werden, dass Attributkombinationen abgefragt werden, die in der Realität nicht existieren, um einen möglichst großen Bereich an Ausprägungen abzufragen (vgl. Matyas \& Kamargianni 2017). SP-Experimente gehen somit als Untersuchungsdesign über die einfache Erhebung von RP hinaus, da mehrdimensionale Einflussfaktoren analysiert werden können (vgl. Hainmueller et al. 2014).

RP- und SP- Experimente können auch kombiniert durchgeführt werden (vgl. Ben-Akiva et al. 1994). So befragten Kagerbauer et al. (2015) in ihrem Projekt „Intermodales Elektromobilitätsmanagement" etwa 170 Personen zunächst mittels RP zu zurückgelegten Wegen und Etappen. Anschließend erfolgte die Konfrontation mit einer hypothetischen Alternative für die berichteten Wege, die ein neues Mobilitätsangebot enthielt, um so die Akzeptanz des Angebots zu ermitteln.

Aus technischer Sicht bildeten neben Befragungen - die aufgrund der geringen Wiederholungsfrequenz schnell veralten und obsolet werden (vgl. Willumsen 2021) - stationäre Zählstellen und manuelle Zählungen lange den Status quo der Datenquellen (vgl. Graf et al. 2019). Da diese allerdings entweder nicht flächendeckend oder nur zeitpunktspezifisch vorhanden waren, wurden verschiedene digitale Tools entwickelt, um Verkehrsmengen ableiten zu können.
So existieren bspw. für den Radverkehr Routing-Apps wie "Strava" und „Komoot", die mittels Crowdsourcing Daten sammeln (vgl. bspw. Francke et al. 2018). CrowdsourcingDaten gehören zu den neuartigen Datenquellen (wie Smartphone-, Fahrkarten-, App-, GPS- und Bluetooth- oder WifiDaten), die es ermöglichen, eine vergleichsweise größere Stichprobe und längere Zeiträume zu erheben (vgl. Willumsen 2021). In diesem Kontext hat das Forschungsprojekt „Movebis“ der TU Dresden Daten aus der vom „Klima-Bündnis" durchgeführten Initiative "Stadtradeln" visualisiert und für Kommunen aufbereitet (vgl. movebis 2021). Auch Sharing-Anbieter nutzen Routing-Daten, um die Standorte der Fahrzeuge zu optimieren (vgl. Wielinski 2018). Um Befragungs- und Crowdsourcing-Data kombiniert nutzen zu können, wird ihre Verknüpfung auch in der Forschung thematisiert (vgl. Baur 2020 sowie Bonnel \& Munizaga 2018).

Um die Nutzung und Präferenzen im Hinblick auf neue Mobilitätsangebote abschätzen zu können, ist ein Multimethodenansatz als Kombination aus Befragungs- mit Crowdsourcing-Daten wie GPS-Trackings sinnvoll. Beispielsweise stellen Danaf et al. (2019) sowie Matyas \& Kamargianni (2017) entsprechende Untersuchungsdesigns vor:

\section{Anmeldung in einer App mit Angabe soziodemographischer Daten sowie Befragung zu Einstellungen und Motiven}

2. Wegetagebuch über GPS-Tracking mit anschließender Validierung der aufgezeichneten Wege sowie Ergänzung der Wegezwecke (RP)

3. Auswahl einer zurückgelegten Route als Referenzsituation für SP-Experiment

\section{Kalibrierung von hypothetischen Alternativsituationen} basierend auf der Referenzsituation

\section{Entscheidung des Befragten zwischen Referenz- und Alternativsituationen}

Durch diesen Multimethodenansatz können RP und SP in Kombination erfasst werden. Zusätzlich können aufgrund des Trackings Routenpräferenzen, Geschwindigkeitsverteilungen sowie konkrete Verhaltenssituationen u. a. an Kreuzungen ausgewertet werden. Diese Informationen können mithilfe der GPS-Koordinaten und des Zeitstempels mit anderen Datenquellen verknüpft werden, um die Topographie, das Wetter, die Verkehrsdichte mit Kfz sowie Points of Interest (Geschäfte, Bildungseinrichtungen, Freizeitorte etc.) in die Analysen einzubeziehen.

Ein inhärenter Nachteil von Tracking-Daten ist jedoch, dass diese in der Regel nicht repräsentativ bzgl. einer interessierenden Grundgesamtheit sind. Die App-Nutzung ist freiwillig 
und es wäre nicht plausibel anzunehmen, dass die Nutzung zufällig erfolgt und somit Zufallsstrichproben einer interessierenden Grundgesamtheit erzeugt werden. So ergibt sich aus der variierenden Internetnutzung, dem sog. „digital divide", eine Überrepräsentation bestimmter Gruppen (vgl. Weber \& Bauder 2013). Größere Verzerrungen können vermieden werden, wenn Tracking-Daten mit Befragungen zu soziodemographischen Variablen kombiniert und gewichtete statistische Analysen durchgeführt werden, die unterrepräsentierte Gruppe hoch- bzw. überrepräsentierte Gruppe heruntergewichten (vgl. bspw. Willumsen 2021). Wenn man auf gewichtete Analysen verzichten möchte, kann durch die Erhebung der soziodemographischen Variablen zumindest ein Eindruck über das Ausmaß an (Nicht-)Repräsentativität gewonnen werden. Als Referenzgröße können dafür die existierenden Befragungen wie MiD und SrV genutzt werden, da diese aufgrund ihres Umfangs erlauben, die quantitative Bedeutung demographischer Gruppen unter den Verkehrsteilnehmenden abzuschätzen.

\section{Zusammenfassung und Schlussfolgerung}

Dieser Artikel diskutiert die Möglichkeiten und Grenzen der Analyse neuer Mobilitätsangebote mittels existierender Befragungen und neuartiger Erhebungsmethoden. Dazu werden zunächst neue Mobilitätsangebote genannt, die zum großen Teil auf Sharing-Konzepten beruhen, eine Schnittmenge mit konventionellen Verkehrsmitteln aufweisen und getrieben durch die Digitalisierung datenbasiert funktionieren. Dazu zählen E-Tretroller, E-Scooter und (E-) Bikes, Car-Sharing, Car-Pooling, Rideselling und öffentliche Bedarfsverkehre. Einhergehend mit den neuen Mobilitätsangeboten werden relevante Forschungsfragen beleuchtet, um zum einen Determinanten des Mobilitätsverhaltens unter Berücksichtigung neuer Mobilitätsangebote abzuleiten und diese zum anderen bei der Integration in Verkehrsmodellen zu berücksichtigen. Da bestehende Mobilitätsbefragungen sowohl in der Verkehrsplanung als auch in der Forschung als Datenbasis für die Analyse und Modellierung dienen, werden anschließend die verschiedenen Adressaten der Befragungen dargestellt, bevor in einer vergleichenden Analyse bestehende Befragungen mit Mobilitätsfokus gegenübergestellt werden.

Die vergleichende Analyse basiert auf der Arbeit von Lanzendorf \& Schönduwe (2018), aktualisiert teilweise verwendete Vergleichsdimensionen auf die letzte Befragungswelle und erweitert die Analyse um neue Mobilitätsangebote und weitere Vergleichsdimensionen. Darüber hinaus wird die Befragung Fahrradmonitor ergänzend gegenübergestellt, um „weiche“ Faktoren zu berücksichtigen. Als Resultat wird im Vergleich der soziodemographischen Angaben deutlich, dass nur die Befragung MiD den Migrationshintergrund erhebt. Darüber hinaus wird in allen untersuchten
Befragungen nur das biologische Geschlecht mit der Auswahl zwischen „männlich“ und „weiblich" erfragt; die Angabe "divers" war in der letzten Welle der Befragungen nicht möglich. Bei der Erfassung von Daten zur üblichen Mobilität und Verkehrsmittelnutzung werden unterschiedliche thematische Schwerpunkte bei den Befragungen deutlich: Während MiD, SrV und MOP den Kfz-Verkehr als Hauptaugenmerk betrachten, fokussiert der Fahrradmonitor auch „weiche“ Faktoren zum Radverkehr. Der Fußverkehr ist in den Befragungen vergleichsweise unterrepräsentiert. Einen Weg koppeln die Befragungen mit Wegetagebuch (MiD, MOP, SrV) immer an einen Wegezweck. Wege, die zu mehreren Zwecken zurückzulegt werden, können nicht korrekt erhoben werden.

Basierend auf der vergleichenden Analyse veranschaulicht dieser Beitrag Lücken anhand zweier Beispiele. Erstens werden neue Mobilitätsangebote im Erhebungsdesign der Befragungen nur punktuell berücksichtigt. So werden im MiD, MOP und SrV Car- und Bikesharing bei der üblichen Verkehrsmittelnutzung zum Teil angeschnitten, allerdings wird die Konsistenz zu den Antwortmöglichkeiten im Wegetagebuch nicht vollständig aufrechterhalten. Allein der Fahrradmonitor geht als Befragung mit einem abweichenden thematischen Fokus vereinzelt auf neue Mobilitätsangebote im Bereich Radverkehr und elektrische Kleinstfahrzeuge ein. Zweitens können Lücken bei der Erfassung „weicher" Faktoren wie Einstellungen und Motiven festgestellt werden. Während beim MiD durch Module vereinzelte Vertiefungsmöglichkeiten geschaffen wurden, sind sowohl beim MOP als auch SrV (nahezu) keine Fragen zu Einstellungen und Motiven vorgesehen. Der Fahrradmonitor beinhaltet hingegen im Rahmen der thematischen Orientierung am Radverkehr eine Vielzahl von Fragen zu „weichen“ Faktoren.

Um die in den Befragungsdaten vorliegenden Lücken zu schließen, sind moderne Forschungsansätze zur kombinierten Erhebung von revealed und stated preferences erforderlich. Um neue Mobilitätsangebote analysieren zu können, wird ein Multimethodenansatz als Kombination aus Befragungsdaten mit Crowdsourcing-Daten wie GPS-Trackings empfohlen. In einer App können Nutzende tatsächlich durchgeführte Wege aufzeichnen lassen und werden anschließend zu einer hypothetischen Situation befragt, in der ein alternatives neues Mobilitätsangebot für einen Teil der Strecke vorgeschlagen wird. Die Integration weiterer Befragungsinhalte zur Erfassung demographischer als auch „weicher" Faktoren wird ebenfalls thematisiert. Die fehlende Repräsentativität von Trackingdaten wird als inhärenter Nachteil aufgezeigt und gewichtete Analysen empfohlen, um Verzerrungen durch über- bzw. unterrepräsentierte Gruppen zu reduzieren. Die Berücksichtigung von Tracking in Kombination mit Befragungsdaten ermöglicht so eine fortlaufende Analyse der dynamischen Entwicklung neuer Mobilitätsangebote am aktuellen Rand. 


\section{Literaturverzeichnis}

Baier, R., Göbbels, A., \& Klemps-Kohnen, A. (2013). Sicherheitskenngrößen für den Radverkehr, Berichte der Bundesanstalt für Strassenwesen - Verkehrstechnik (V); 228, Fachverlag NW in Carl Ed. Schünemann KG, Bremen.

Baier, R. (2009). Das Handbuch für die Bewertung der Verkehrssicherheit von Strassen. Deutscher Straßen- und Verkehrskongress Düsseldorf 2008 (FGSV 001/22).

Barillère-Scholz, M., Büttner, C., \& Becker, A. (2020). Mobilität 4.0: Deutschlands erste autonome Buslinie in Bad Birnbach als Pionierleistung für neue Verkehrskonzepte. In Autonome Shuttlebusse im ÖPNV (pp. 15-22). Springer Vieweg, Berlin, Heidelberg.

Baur, S. (2020). Erschließung von Crowd Data und Verknüpfung mit Befragungsdaten im Bereich Verkehr. Stadtforschung und Statistik: Zeitschrift des Verbandes Deutscher Städtestatistiker, 33(2), 30-35.

Ben-Akiva, M., Bradley, M., Morikawa, T., Benjamin, J., Novak, T., Oppewal, H., \& Rao, V. (1994). Combining revealed and stated preferences data. Marketing Letters, 5(4), 335-349.

Bierlaire, M., Chen, J., \& Newman, J. (2010). Modeling route choice behavior from smartphone GPS data. Report TRANSPOR.

Blechschmidt, A., Schönduwe, R., \& Lanzendorf, M. (2005). Nutzungsmöglichkeiten von Mobilitätsdaten in der Region Frankfurt Rhein-Main. Regionale Mobilitätserhebungen und Mobilitätskennziffern im Vergleich - Eine Handreichung für die Praxis. Arbeitspapiere zur Mobilitätsforschung Nr. 4. Frankfurt a.M.

Bonnel, P., \& Munizaga, M. A. (2018). Transport survey methods in the era of big data facing new and old challenges. Transportation Research Procedia, 32, 1-15.

Borgstedt, S., Hecht, J., \& Jurczok, F. (2018). Fahrrad-Monitor 2017. Ergänzung. Fahrradstraßen, Fahrrad-Pendeln und Radschnellwege. Ergebnisse einer repräsentativen Online-Befragung. Version vom 13.04. 2018. https://repository.difu.de/jspui/bitstream/difu/249748/1/DS1801.pdf, (Letzter Abruf: 21.07.2021).

Bracher, T. (2016). Fahrrad- und Fußverkehr: Strukturen und Potentiale. In Handbuch Verkehrspolitik (pp. 265-291). Springer VS, Wiesbaden.

Cyganski, R., \& Justen, A. (2007). Maßnahmensensitive Nachfragemodellierung in mikroskopischen Personenver- kehrsmodellen. DVWG Jahresband 2007: Ausgewählte Beiträge der zentralen wissenschaftlichen Veranstaltungen, (B 314), 168-183, Stuttgart.

Dallmeyer, J. (2014). Simulation des Straßenverkehrs in der Großstadt: das Mit- und Gegeneinander verschiedener Verkehrsteilnehmertypen. Springer-Verlag, Berlin, Heidelberg.

Danaf, M., Atasoy, B., De Azevedo, C. L., Ding-Mastera, J., Abou-Zeid, M., Cox, N. \& Ben-Akiva, M. (2019). Contextaware stated preferences with smartphone-based travel surveys. Journal of choice modelling, 31, 35-50.

Deffner, J. (2018). Fuß- und Radverkehr. In Verkehrspolitik (pp. 415-444). Springer VS, Wiesbaden.

Dell'olio, L., Ibeas, A., de Oña, J., \& de Oña, R. (2018). Designing a survey for public transport users. Public Transp. Qual. Serv, 49-61.

Difu-Berichte (2021). Was ist eigentlich ... Mikromobilität?. Difu-Berichte Heftarchiv, Bd. 2. Deutsches Institut für Urbanistik (Difu), Berlin.

Döring, T., \& Aigner-Walder, B. (2017). Verkehrs-, umweltund raumbezogene Aspekte der Elektromobilität aus der Sicht des Nutzerverhaltens. Raumforschung und Raumordnung-Spatial Research and Planning, 75(4), 339-353.

Ecke, L., Chlond, B., Magdolen, M., \& Vortisch, P. (2020). Deutsches Mobilitätspanel (MOP) - Wissenschaftliche Begleitung und Auswertungen Bericht 2019/2020: Alltagsmobilität und Fahrleistung. Institut für Verkehrswesen (KIT). Karlsruhe.

Eggs, J., Follmer, R., Gruschwitz, D., Nobis, C., Bäumer, M., \& Pfeiffer, M. (2018). Mobilität in Deutschland - MiD: Methodenbericht. Studie von infas, DLR, IVT und infas $360 \mathrm{im}$ Auftrag des BMVI. Bonn, Berlin.

Follmer, R. (2019). Mobilität in Deutschland - MiD: Wegeerfassung im Etappenkonzept. Studie von infas, DLR, IVT und infas $360 \mathrm{im}$ Auftrag des BMVI. Bonn, Berlin.

Francke, A., Becker, T., \& Lißner, S. (2018). Big Data im Radverkehr. Ergebnisbericht "Mit Smartphones generierte Verhaltensdaten im Radverkehr". Dresden.

Friedrich, M. (2011). Wie viele? Wohin? Womit? Was können uns Verkehrsnachfragemodelle wirklich sagen. Tagungsbericht Heureka, 11, FGSV Verlag, Köln.

Gerike, R., Hubrich, S., Ließke, F., Wittig, S., \& Wittwer, R. (2020). Sonderauswertung zum Forschungsprojekt „Mobili- 
tät in Städten - SrV 2018 “, Dresden.

Götz, K., Deffner, J., \& Klinger, T. (2016). Mobilitätsstile und Mobilitätskulturen - Erklärungspotentiale, Rezeption und Kritik. In Handbuch Verkehrspolitik (pp. 781-804). Springer VS, Wiesbaden.

Götz, J., \& Stein, M. (2018). Freizeitmobilität und -verkehr. In Verkehrspolitik (pp. 323-346). Springer VS, Wiesbaden.

Graf, L., Pucher, G., \& Gatscha, S. (2019). Ersatzwertbildung für stationäre Verkehrsdetektoren. AGIT: Journal für Angewandte Geoinformatik, (5), 184-191.

Hagen, T., M. Sunder, E. Lerch u. S. Saki (2021): Effekte der COVID-19-Pandemie auf Mobilität und Verkehrsmittelwahl. In: Straßenverkehrstechnik 1/2021, S. 7-14.

Hainmueller, J., Hopkins, D. J., \& Yamamoto, T. (2014). Causal inference in conjoint analysis: Understanding multidimensional choices via stated preference experiments. Political analysis, 22(1), 1-30.

Hellenschmidt, J., \& Wittwer, R. (2007). Wenn zwei das Gleiche tun, erheben sie noch lange nicht dasselbe. Die Haushaltsbefragungen" Mobilität in Deutschland-MiD" und" Mobilität in Städten-SrV" stehen vor ihrer Neuauflage/If two do the same thing, they do not collect the same roughly. Internationales Verkehrswesen, 59(6), 270-274.

Hubrich, S., Ließke, F., Wittwer, R., Wittig, S., \& Gerike, R. (2019). Methodenbericht zum Forschungsprojekt.,,Mobilität in Städten - SrV 2018 ", Dresden.

Kagerbauer, M., Schröder, J. O., Weiß, C., \& Vortisch, P. (2015). Intermodale Mobilität. In Entscheidungen beim Übergang in die Elektromobilität (pp. 567-583). Springer Gabler, Wiesbaden.

Kantar (2020). Deutsches Mobilitätspanel (MOP) - Erhebung der Alltagsmobilität sowie der Pkw-Fahrleistungen und Kraftstoffverbräuche Endbericht zum Paneljahr 2019/2020, München. https://mobilitaetspanel.ifv.kit.edu/downloads/ KANTAR_Endbericht_MOP_2019_2020_inkl_Anlagen.pdf (Abruf am 28.06.2021).

Klinger, T., Deffner, J., Kemen, J., Stein, M., \& Lanzendorf, M. (2016). Sharing-Konzepte für ein multioptionales Mobilitätssystem in FrankfurtRheinMain. Analyse neuerer Entwicklungen und Ableitung von Handlungsoptionen für kommunale und regionale Akteure. Im Auftrag des HMWEVL. Schlussbericht. Arbeitspapiere zur Mobilitätsforschung, (9).

Knapp, F. D. (2015). Determinanten der Verkehrsmittelwahl (Vol. 10). Duncker \& Humblot, Berlin.
Lange, P., \& Malik, J. (2019). Radschnellverbindungen. Leitfaden zur Potenzialanalyse und Nutzen-Kosten-Analyse. https://repository.difu.de/jspui/bitstream/difu/257038/1/ DS2196.pdf, (Letzter Abruf: 21.07.2021).

Lanzendorf, M., \& Hebsaker, J. (2017). Mobilität 2.0 - Eine Systematisierung und sozialräumliche Charakterisierung neuer Mobilitätsdienstleistungen. In Verkehr und Mobilität zwischen Alltagspraxis und Planungstheorie (pp. 135-151). Springer VS, Wiesbaden.

Lanzendorf, M., \& Schönduwe, R. (2018). Datenerhebung zur Erfassung des Mobilitätsverhaltens. 3.2.1.2-Handbuch der kommunalen Verkehrsplanung (pp. 1-24), 80. Ergänzungs-Lieferung 2/2018. Berlin, Bonn.

Leihs, D., Siegl, T., \& Hartmann, M. (2014). Makroskopische Modellierung. In City-Maut (pp. 149-169). Springer Vieweg, Wiesbaden.

Leszczynski, T. (2018). „Sensing Comfort “ - der Einfluss von Fahrbahnoberflächen auf den objektiven Fahrkomfort während des Fahrradfahrens. Doctoral dissertation, Wien.

Löwa, S., \& Gertz, C. (2017). Praktische Erfahrungen beim Einsatz von Verkehrsmodellen in Kommunen. ECTL Working Paper 48, Hamburg.

Matyas, M., \& Kamargianni, M. (2017). A stated preference experiments for mobility-as-a-service plans. In 2017 5th IEEE International Conference on Models and Technologies for Intelligent Transportation Systems (MT-ITS) (pp. 738-743). IEEE.

Movebis (2021). https://www.movebis.org/das-projekt/ (Letzter Abruf: 21.07.2021).

Nationaler Radverkehrsplan (2019). nrvp.de/21127 (Letzter Abruf: 21.07.2021).

Nobis, C., \& Kuhnimhof, T. (2018). Mobilität in Deutschland - MiD: Ergebnisbericht. Studie von infas, DLR, IVT und infas $360 \mathrm{im}$ Auftrag des BMVI. Bonn, Berlin.

Oostendorp, R., Oehlert, J., \& Heldt, B. (2020). Neue Mobilitätsangebote in Wohnquartieren: Maßnahmen und Wirkungen aus Sicht von öffentlicher Verwaltung, Wohnungswirtschaft und Planung. In Mobilität, Erreichbarkeit, Raum (pp. 179-200). Springer VS, Wiesbaden.

PTV/Fraunhofer ISI/M-Five (2019). Verlagerungswirkungen und Umwelteffekte veränderter Mobilitätskonzepte im Personenverkehr. Wissenschaftliche Beratung des BMVI zur Mobilitäts- und Kraftsstoffstrategie, Karlsruhe. https:// www.bmvi.de/SharedDocs/DE/Anlage/G/MKS/studie-ver- 
lagerungswirkungenumwelteffekte-mobilitaetskonzepte. pdf?_blob=publicationFile (Abruf am 21.07.2021).

Schad, H., Wegelin, P., Mahrer, M., Marconi, D., Pfund, S., \& Lutzensberger, M. (2020). Einflussfaktoren auf Alltagsmobiliät und nicht-alltägliche Mobilität. Eidgenössisches Departement für Umwelt, Energie und Kommunikation UVEK, Bundesamt für Strassen.

Stiewe, M., \& Krause, J. (2012). Geschlechterverhältnisse und Mobilität - Welchen Beitrag leisten Mobilitätserhebungen? In M. Schrenk, V. V. Popovic, P. Zeile \& P. Elisei (Hrsg.), Tagungsband Zur Real Corp 2012 (S. 321-330). Schwechat.

Vrtic, M. (2004) Ein hierarchisches („Nested“) Logit-Modell für die Analyse kombinierter Stated- und Revealed-Preference-Daten zur Verkehrsmittelwahl, Vortrag, 12 DVWGWorkshop „Statistik und Verkehr" 2004, Mann-heim.

Weber, H. J. L., \& Bauder, M. (2013). Neue Methoden der Mobilitätsanalyse: Die Verbindung von GPS-Tracking mit quantitativen und qualitativen Methoden im Kontext des Tourismus. Raumforschung und Raumordnung, 71(2), 99113.

Wermuth, M., Sommer, C., \& Wulff, S. (2006). Erhebung der individuellen Routenwahl zur Weiterentwicklung von Umlegungsmodellen. in: Bundesanstalt für Straßenwesen, Reihe Verkehrstechnik, Heft V. Fachverlag NW in Carl Ed. Schünemann KG, Bremen.

Wessel, J. (2020). Using weather forecasts to forecast whether bikes are used. Transportation research part A: policy and practice, 138, 537-559.

Wielinski, G., Trépanier, M., Morency, C., \& Habib, K. N. (2018). Comparing multiple data streams to assess freefloating carsharing use. Transportation Research Procedia, 32, 617-626.

Willumsen, L. (2021). Use of Big Data in Transport Modelling. International Transport Forum Discussion Papers, No. 2021/05, OECD Publishing, Paris.

Zhao, F., Pereira, F. C., Ball, R., Kim, Y., Han, Y., Zegras, C., \& Ben-Akiva, M. (2015). Exploratory analysis of a smartphonebased travel survey in Singapore. Transportation Research Record, 2494(1), 45-56.

ZIV (2021). https://www.ziv-zweirad.de/fileadmin/redakteure/Downloads/Marktdaten/PM 2021 10.03. ZIVPraesentation 10.03.2021 mit_Text.pdf (Letzter Abruf: 21.07.2021).

\section{AutorInnenangaben}

\section{Prof. Dr. Tobias Hagen}

Direktor Research Lab for Urban Transport, Professor für Volkswirtschaftslehre und Quantitative Methoden, Frankfurt University of Applied Sciences

Frankfurt University of Applied Sciences

Nibelungenplatz 1

60318 Frankfurt am Main

E-Mail: thagen@fb3.fra-uas.de

\section{M.Sc. Nicole Reinfeld}

Wissenschaftliche Mitarbeiterin, Research Lab for Urban Transport

Frankfurt University of Applied Sciences

Nibelungenplatz 1

60318 Frankfurt am Main

E-Mail: nicole.reinfeld@fb1.fra-uas.de 